\title{
Dust temperature maps of the Galactic plane: The Herschel spectral energy distribution fitting with Cloudy predictions ${ }^{\star}, \star \star$
}

\author{
Jiali Zhu ${ }^{1,2}$ and Maohai Huang ${ }^{1}$
}

\author{
1 National Astronomical Observatories, 20A Datun Road, Chaoyang District, 100012 Beijing, PR China \\ e-mail: mhuang@nao.cas.cn \\ 2 University of Chinese Academy of Sciences, 100049 Beijing, PR China
}

Received 21 September 2013 / Accepted 11 February 2014

\begin{abstract}
Context. Dust grains absorb the interstellar far ultra-violet and visible photons and re-emit them in far-infrared (FIR) wavebands. The dust FIR continuum can be predicted by a grid of models using various values of the interstellar radiation field.

Aims. We analyze the dust continuum emission in two Hi-GAL science-demonstration phase (SDP) fields using both the radiative transfer code, Cloudy, and the DustEM dust model, to explore the effect of radiative transfer on dust temperature. The $500 \mu \mathrm{m}$ submillimeter excess emission and the very small grain (VSG) contribution to the $70 \mu \mathrm{m}$ intensity are investigated by spectral energy distribution (SED) fitting using the Cloudy model.

Methods. By comparing the observation with the model prediction, we derive dust temperature maps of the two SDP fields by fitting the dust SED with 4-band data (SPIRE bands plus PACS $160 \mu \mathrm{m}$ ) using both Cloudy and DustEM models. Considering radiative transfer and grain physics simultaneously, we investigate the existence of a $500 \mu \mathrm{m}$ excess and estimate the VSG contribution to the $70 \mu \mathrm{m}$ intensity by fitting the dust SED with 3-band data $(160,250$, and $350 \mu \mathrm{m})$ and 5-band data (SPIRE and PACS bands), respectively.

Results. We confirm that the field with star formation activities have a higher temperature $(18.7 \pm 0.9 \mathrm{~K})$ than the quiescent region $(15.2 \pm 0.6 \mathrm{~K})$. We find that the radiative transfer affects the FIR SED of the SDP fields and results in a higher temperature distribution than the dust-only model fit. There is no significant detection of a $500 \mu \mathrm{m}$ excess in the two SDP fields. The relative contribution from the VSGs to the $70 \mu \mathrm{m}$ intensity can be up to $50 \%$.
\end{abstract}

Key words. ISM: general - dust, extinction - infrared: ISM - methods: data analysis

\section{Introduction}

Interstellar dust particles are size distributed. Mathis et al. (1977) developed a power-law grain-size distribution on the basis of the observed interstellar extinction from infrared to ultraviolet wavelengths: $n(a) \propto a^{-3.5}$, where $n(a)$ is the number density of grains with radius $<a(0.005 \mu \mathrm{m}<a<0.25 \mu \mathrm{m})$. The emission from dust grains with different sizes dominates farinfrared (FIR) emission in different wavelength ranges. Small grains $(\lesssim 0.005 \mu \mathrm{m})$ radiate at $\lambda \lesssim 50 \mu \mathrm{m}$ by cooling after they are heated by single starlight photons (Draine 2003). Big grains (BGs; $\gtrsim 0.01 \mu \mathrm{m}$ ) are at thermal equilibrium, emitting FIR continuum at $\lambda \gtrsim 50 \mu \mathrm{m}$.

To derive the equilibrium temperature of BGs in the interstellar radiation field (ISRF), there have been mainly two methods to analyze FIR spectral energy distributions (SED) of dust grains. One is fitting the SED with a modified blackbody (i.e., gray body; e.g., Paradis et al. 2010). The other is fitting the SED with FIR continuum emission calculated by dust models, such as DustEM (Compiègne et al. 2010).

\footnotetext{
* Herschel is an ESA space observatory with science instruments provided by European-led Principal Investigator consortia and with important participation from NASA.

$\star \star$ Reduced Herschel maps (FITS) are only available at the CDS via anonymous ftp to cdsarc.u-strasbg. fr $(130.79 .128 .5)$ or via http://cdsarc.u-strasbg.fr/viz-bin/qcat?J/A+A/564/A111
}

In the gray body method, the temperature of big grains is approximated by

$F_{v} \propto \lambda^{-\beta} B_{v}\left(T_{\text {dust }}\right)$

where $F_{v}$ is the observed flux, $B_{v}$ is the blackbody emission, $T_{\text {dust }}$ is dust temperature, and $\beta$ is the emissivity spectral index that modifies the blackbody emission. Boulanger et al. (1996) found that $\beta=2$ for the solar neighborhood derived from diffuse FIR emission, which is measured by Diffuse InfraRed Background Experiment (DIRBE) and Far InfraRed Absolute Spectrophotometer (FIRAS) at high Galactic latitudes, while Paradis et al. (2010) showed variations of the spectral emissivity spectral index in the range $1.8 \sim 2.6$ for the Galactic plane. This method assumes that the dust abundance does not vary and dust grains are single size grains.

In the second method, DustEM is used to predict the emission of dust grains with specified grain types and grain size distributions. This method is more physical than using a gray body fit, since it considers the grain size distribution. Bernard et al. (2010) derived dust temperature maps of the Galactic plane using the DustEM code as a modeling tool. The DustEM code does not include any radiative transfer calculation. For dust and gas near hot OB stars, it is more physical to consider both the gas and grain absorption of radiation. To do that, calculation of full radiative transfer is needed.

The Cloudy code (Ferland et al. 2013) includes grain models into self-consistent radiative transfer calculation, which 
produces the continuum and line emission of gas and dust in equilibrium condition. In this paper, we compare the predicted dust emission of Cloudy models for various values of ISRF with Hi-GAL (Molinari et al. 2010) science-demonstration phase (SDP) data to derive dust temperature maps of SDP fields. The SDP fields are two $2^{\circ} \times 2^{\circ}$ images centered at $l=30^{\circ}, b=0^{\circ}$, and $l=59^{\circ}, b=0^{\circ}$.

We introduce the observation data and analysis method in Sect. 2. Results of 4-band $(160,250,350$, and $500 \mu \mathrm{m})$ fits and the comparison between Cloudy and DustEM results are presented in Sect. 3. In Sect. 4, we present the results of 3-band $(160,250$, and $350 \mu \mathrm{m})$ fits for Cloudy models and the calculation of the $500 \mu \mathrm{m}$ excess. The contribution from very small grains to the $70 \mu \mathrm{m}$ intensity is estimated in Sect. 5. We summarize in Sect. 6.

\section{The observation and analysis method}

The Herschel (Pilbratt et al. 2010) Hi-GAL images provide data with extraordinary spatial, wavelength, and dynamic range coverage for studying the FIR emission of dust grains in the Galactic plane. The Hi-GAL SDP fields are products of the Hi-GAL program, which are observed with PACS (Poglitsch et al. 2010) $70 \mu \mathrm{m}$ and $160 \mu \mathrm{m}$ channels and SPIRE (Griffin et al. 2010) 250, 350, and $500 \mu \mathrm{m}$ channels on board Herschel. The Hi-GAL data processing and map making are described in Molinari et al. (2010). Since the GLS map-making technique is known to introduce artifacts in the maps, namely crosses and stripes in correspondence of the brightest sources, a weighted post-processing of the GLS maps (WGLS, Piazzo et al. 2012) has been applied to finally obtain images in which artifacts are removed or heavily attenuated. The SDP images used in this article are the latest version released to the Hi-GAL consortium. The data have been absolutely calibrated. For more information of the absolute calibration of the Hi-GAL pipeline, we refer the reader to Elia et al. (2013).

The very small grains (VSGs, radius $<0.005 \mu \mathrm{m}$ ) are stochastically heated. The contribution from the VSGs to the PACS $70 \mu \mathrm{m}$ channel can be up to $\sim 50 \%$ (Compiègne et al. 2010). To focus on equilibrium temperature of BGs, we exclude the $70 \mu \mathrm{m}$ emission in our SED fitting to derive the temperature maps. The adopted absolute calibration uncertainty is $20 \%$ for PACS bands (Bernard et al. 2010) and 15\% for SPIRE bands (Paradis et al. 2010). We exclude the higher latitude areas $\left(|b|>0.9^{\circ}\right)$, where the data could be less reliable. Maps of all five channels are smoothed to the same resolution as SPIRE $500 \mu \mathrm{m}$ channel with the convolution kernels given by Karl Gordon ${ }^{1}$.

We adopt the build-in ISM dust grains and ISM gas abundance of Cloudy. This grain set has two components, graphite and silicate. The size distribution of ISM dust grains is given by Mathis et al. (1977). This grain set generally reproduces the observed overall extinction properties for $R_{V}=3.1$, or the average Milky Way extinction (Fitzpatrick 2004). The size of adopted ISM grains ranges from $0.005 \mu \mathrm{m}$ to $0.25 \mu \mathrm{m}$ with a dust-to-gas mass ratio, $D / G=6.362 \times 10^{-3}$. PAHs are included in our calculation with a power-law size distribution, as described in Abel et al. (2008). The geometry is plane-parallel.

With input parameters specified above, we use Cloudy to pre-calculate the dust FIR SED for the gas column density, $N(\mathrm{H})=10^{20} \mathrm{H} \mathrm{cm}^{-2}$, for various values of ISRF. The SED of ISRF is described in Black (1987). The scaling factor $U$ in

\footnotetext{
1 http://dirty.as.arizona.edu/ kgordon/mips/conv_psfs/ conv_psfs.html
}

Cloudy scales the intensity of ISRF. We have $U=1.04 G_{0}$ in Habing field unit $\left(1.6 \times 10^{-3} \mathrm{erg} \mathrm{cm}^{-2} \mathrm{~s}^{-1}\right.$; Habing 1968). In the calculations, $U$ is set to range from $10^{-2}$ to $10^{2}$ with $0.02 \mathrm{dex}$ steps. The gas density $n$ is fixed at $10 \mathrm{~cm}^{-3}$. We test the effect of gas density by comparing the predicted FIR SED for varying gas density, which ranges from $1 \mathrm{~cm}^{-3}$ to $10^{3} \mathrm{~cm}^{-3}$ with 1 dex steps, when the logarithmic value of $U$ is set to range from -2 to 2 with 1 dex steps. For a certain $G_{0}$, tests show that the difference between the resulted SED of the FIR continuum at $n=10 \mathrm{~cm}^{-3}$ and at other value of gas density is less than $0.2 \%$ when $N(\mathrm{H})$ is fixed to $10^{20} \mathrm{H} \mathrm{cm}^{-2}$. Thus, we ignore the effects of gas density. For every map pixel, we compare the observed fluxes with model predictions by computing a chi-square statistic using equation,

$\chi^{2}=\sum_{i=1}^{N}\left(\frac{F_{\mathrm{obs}, i}-s \times F_{\mathrm{mod}, i}}{\sigma_{i}}\right)^{2}$,

where $F_{\mathrm{obs}, i}$ is the observed flux of waveband $i, F_{\text {mod, } i}$ is the model predicted flux of waveband $i$, and $\sigma_{i}$ is the calibration uncertainty of waveband $i$. In this equation, $s$ is the scaling factor chosen to minimize the $\chi^{2}$ between observation and model prediction. The value $N$ is the number of band, and the degrees of freedom of $\chi^{2}$ distribution is $N-M$, where $M$ is the number of free parameters determining the model prediction. In our case, $G_{0}$ and $s$ are the varying parameters.

We minimize the $\chi^{2}$ to recover the best fit $G_{0}$ for every pixel. The parameter $G_{0}$ determines the SED of dust FIR continuum. We estimate dust temperature using the formula $T_{\mathrm{d}}=$ $17.5 \mathrm{~K}\left(G_{0}\right)^{1 /(4+\beta)}$ (Bernard et al. 2010; Boulanger et al. 1996; Compiègne 2010) with a dust emissivity index $\beta=2$. For both fields, we fit the dust SED with 4-band data using Cloudy. When the scaling factor $s$ is held fixed, we estimate the $68 \%$ confidence interval of $G_{0}$ by increasing the minimized value of $\chi^{2}$ by 1 for every pixel (Avni \& Bahcall 1976).

To explore the effect of radiative transfer, we also fit the SED with 4-band data using the DustEM model. Following the same procedures as 4-band fits for Cloudy models, we first use the DustEM code to pre-calculate the dust FIR SED for various values of ISRF. The SED of ISRF, the dynamic range and the increment step of $G_{0}$, and the grain set used in DustEM models are the same as what we adopt in the SED fitting for Cloudy models. The pre-calculated dust FIR SED is then compared with the observation. We adjust $G_{0}$ and the scaling factor $s$ in Eq. (2) to fit the SED of every map pixel and compute the $\chi^{2}$ using Eq. (2). We find the best fit $G_{0}$ by minimizing the $\chi^{2}$ of every pixel and then estimate dust temperature using the formula $T_{\mathrm{d}}=17.5 \mathrm{~K}\left(G_{0}\right)^{1 /(4+\beta)}$.

To investigate the existence of a $500 \mu \mathrm{m}$ excess, we use the same procedures to fit the SED with 3-band data (160, 250, and $350 \mu \mathrm{m}$ ) using the Cloudy code and compare them to results of 4-band fits for Cloudy models. We also fit the SED with 5-band data using Cloudy to estimate the contribution from the VSGs to the $70 \mu \mathrm{m}$ intensity by comparing the results of 5-band fits with 4-band fits for Cloudy models.

\section{Results of 4-band fits for Cloudy and DustEM models}

In this section, we compare the results for Cloudy models with DustEM models to explore the effects of radiative transfer. We present the temperature maps and $\chi^{2}$ distribution of 4-band fits for Cloudy results in Figs. 1, and 2 and summarize results in Table 1. We show the temperature histograms in Fig. 3. For the 

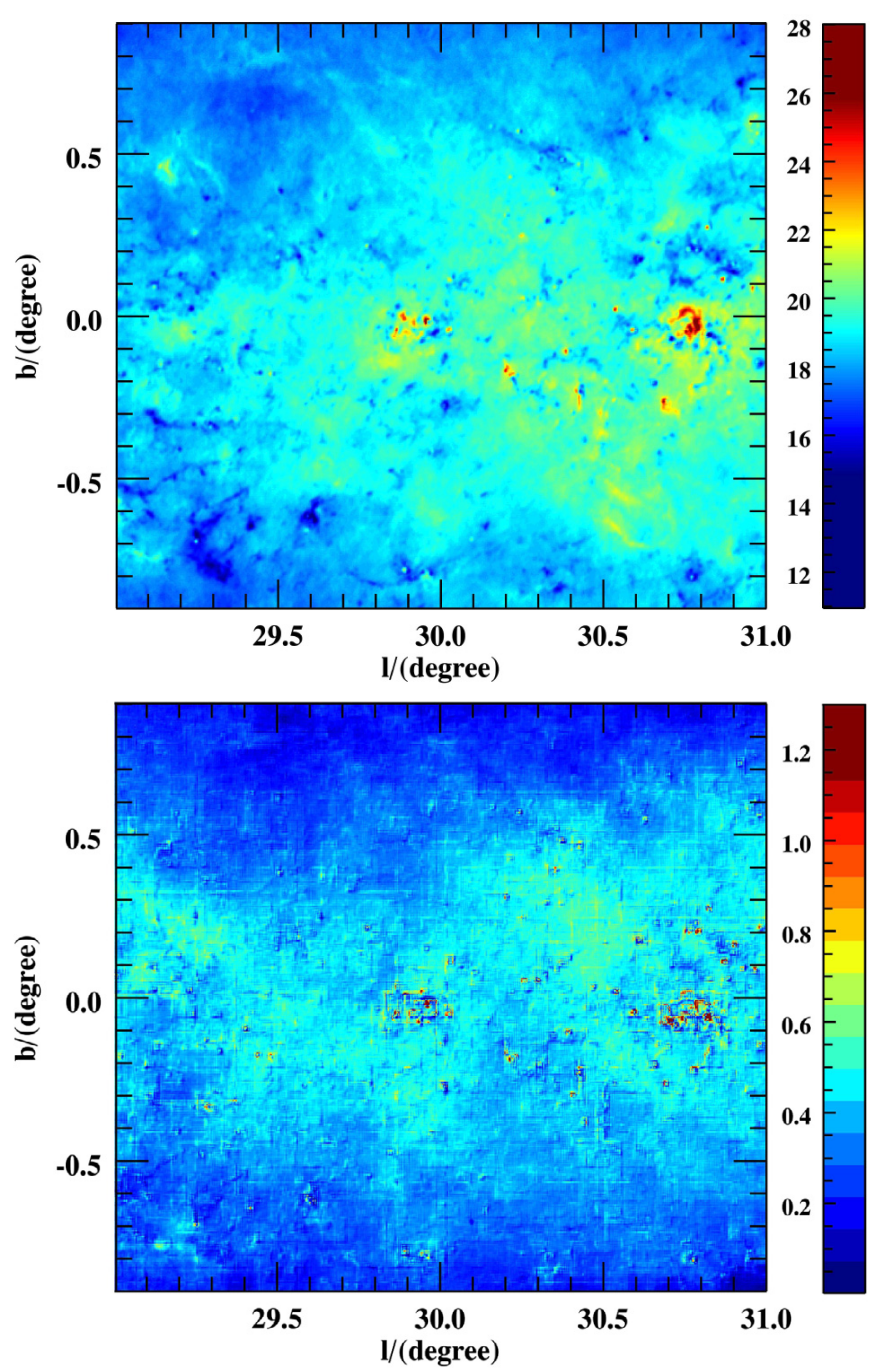

Fig. 1. Temperature maps and $\chi^{2}$ maps of the $l=30^{\circ}$ field. Upper panel: the temperature map. Lower panel: $\chi^{2}$ map.

$l=30^{\circ}$ field, the average temperature is $18.7 \mathrm{~K}$ with the root mean square (RMS) value $0.9 \mathrm{~K}$. For the $l=59^{\circ}$ field, the average temperature is $15.2 \mathrm{~K}$ with the RMS $0.6 \mathrm{~K}$. For both fields, the percentages of pixels with $\chi^{2}>2$ are both $0.015 \%$.

To explore the effects of radiative transfer, we overlay histograms of Cloudy results on DustEM results and present them in Fig. 4. For both fields, the derived maximum and average temperatures of DustEM results (Table 1) are lower than Cloudy results. Comparing histograms in the upper panels of Fig. 4, we find that the temperature distributions of DustEM results shift toward lower value of temperature. The difference between results for Cloudy and DustEM models is better shown in the lower panels of Fig. 4. We plot the temperature of every pixel for Cloudy and DustEM models in the lower panels of Fig. 4 and overlay the $68 \%$ confidence interval of Cloudy results on the two plots. A black dot indicates the temperatures of every pixel for DustEM ( $T_{\text {DustEM }}$, the value of $y$ axis) and Cloudy results $\left(T_{\text {Cloudy }}\right.$, the value of $x$ axis); two red lines indicate the $68 \%$ confidence interval of dust temperature for Cloudy results. A black line of $y=x$ is also plotted. If $T_{\text {Cloudy }}$ equals to $T_{\text {DustEM }}$, the black dot would fall on the black line; if $T_{\text {DustEM }}$ is consistent with $T_{\text {Cloudy }}$ within the $68 \%$ confidence interval, the black dot would fall between the two red lines. As shown in the lower panels of Fig. 4, most black dots fall outside the area between the two red lines. To
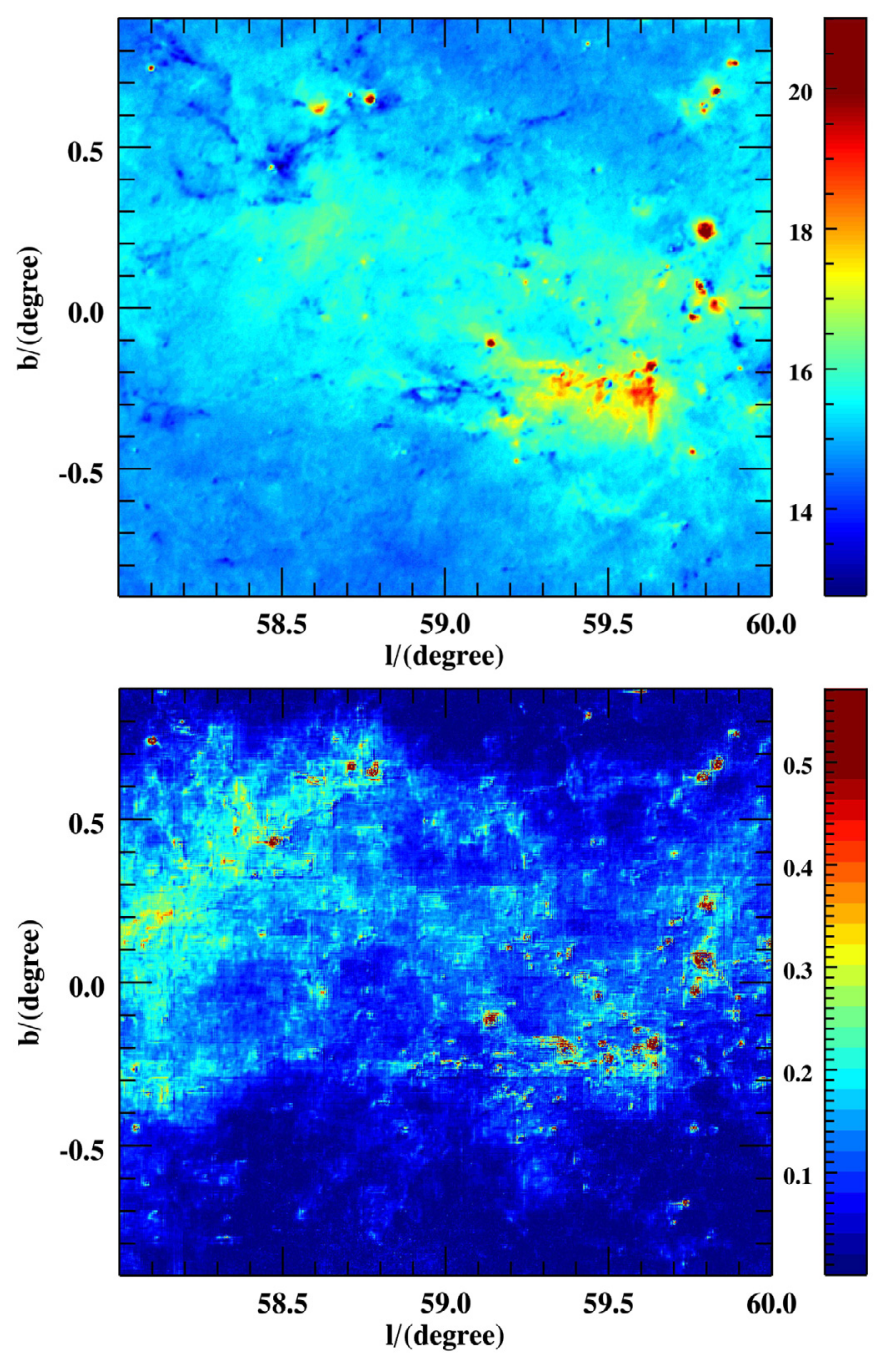

Fig. 2. Temperature maps and $\chi^{2}$ maps of the $l=59^{\circ}$ field. Upper panel: the temperature map. Lower panel: $\chi^{2}$ map.

Table 1. Results of 4-band fits.

\begin{tabular}{lcccc}
\hline \hline Fields & \multicolumn{2}{c}{$l=30^{\circ}$} & \multicolumn{2}{c}{$l=59^{\circ}$} \\
& Cloudy & DustEM & Cloudy & DustEM \\
\hline Average $T_{\text {dust }}(\mathrm{K})$ & $18.7^{a}$ & $17.8^{b}$ & $15.2^{c}$ & $14.7^{d}$ \\
Max $T_{\text {dust }}(\mathrm{K})$ & 32.8 & 29.9 & 29.4 & 26.7 \\
Min $T_{\text {dust }}(\mathrm{K})$ & 10.9 & 10.9 & 12.8 & 12.3 \\
\hline
\end{tabular}

Notes. ${ }^{(a)}$ The RMS is $0.9 \mathrm{~K} .{ }^{(b)}$ The RMS is $0.8 \mathrm{~K} .{ }^{(c)}$ The RMS is $0.6 \mathrm{~K} .{ }^{(d)}$ The RMS is $0.5 \mathrm{~K}$.

find the degree of inconsistency, we count the number of black dots outside the area between the two red lines. For the $l=30^{\circ}$ field, $99.9 \%$ of all pixels for DustEM results are inconsistent with Cloudy results within the $68 \%$ confidence interval. For the $l=59^{\circ}$ field, the temperature of all pixels for DustEM results are inconsistent with Cloudy results within the $68 \%$ confidence interval. This high degree of inconsistency between DustEM and Cloudy results indicates that 1 ) the radiative transfer has an effect on FIR SED compared to predictions using the dust model only; 2) the effect is pronounced in both the hotter region and the colder region and makes the temperature distribution shift toward higher value of temperature. 


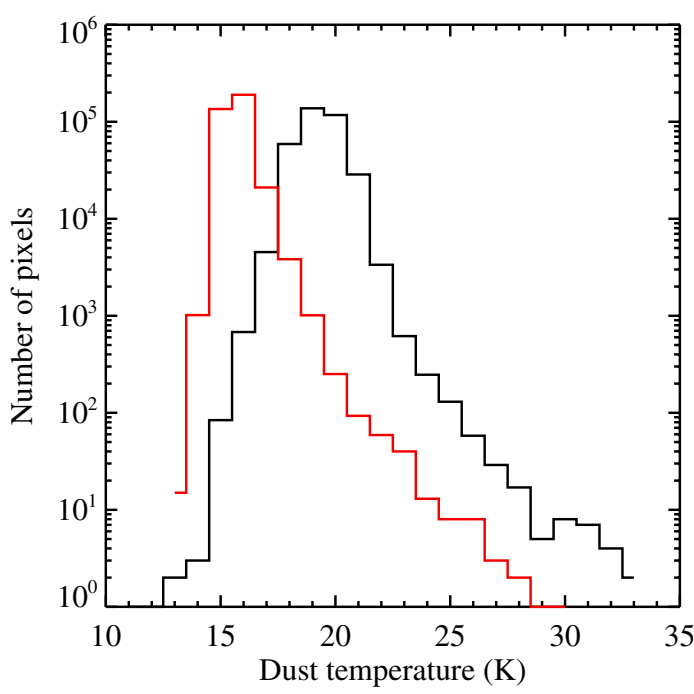

Fig. 3. Temperature histograms for 4-band fits. Black line: the histogram of the $l=30^{\circ}$ field. Red line: the histogram of the $l=59^{\circ}$ field.
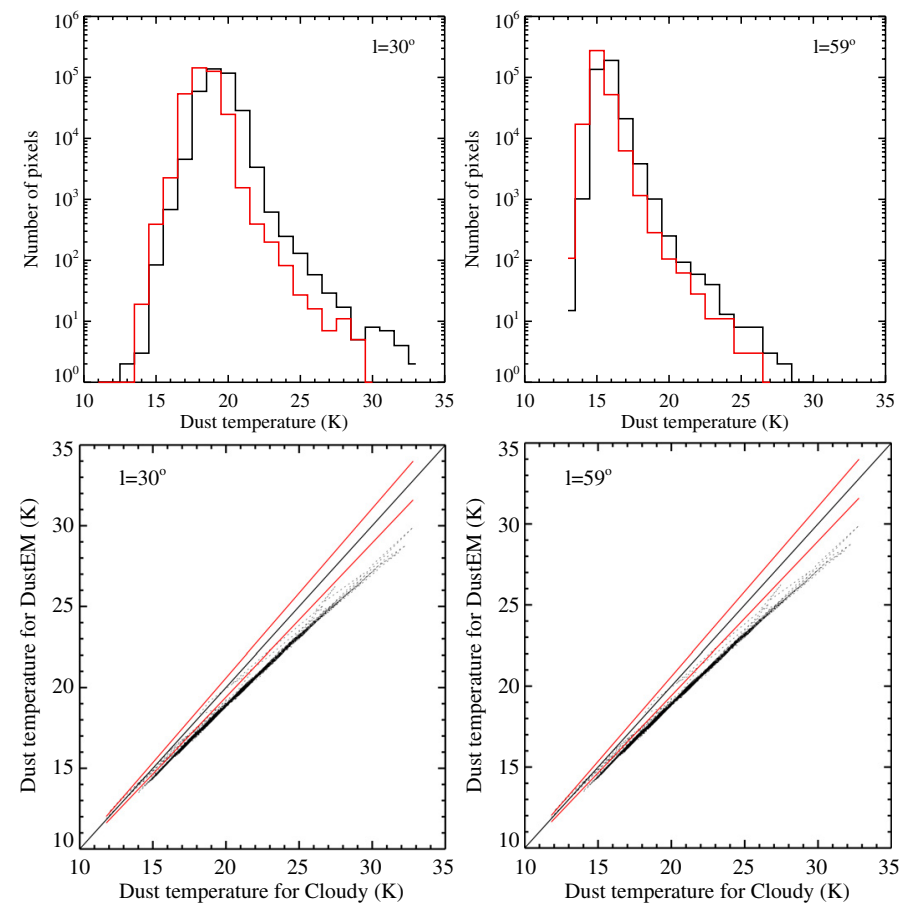

Fig. 4. Comparison of DustEM and Cloudy results. Upper panels: the overlay of histograms; black line is the result of Cloudy; red line is the result of DustEM. Lower panels: the function of the black line: $y=x$; black dot indicates the temperatures of every pixel for DustEM results and for Cloudy results; red lines indicate the $68 \%$ confidence interval of Cloudy results.

\section{Results of 3-band fits for Cloudy models}

Using Cloudy models, we fit the dust SED using 160, 250, and $350 \mu \mathrm{m}$ data to investigate the influence of $500 \mu \mathrm{m}$ data points in the SED fitting and the existence of a $500 \mu \mathrm{m}$ excess.

To investigate the influence of $500 \mu \mathrm{m}$ data point in the SED fitting, we compare the results of 3-band and 4-band fits for Cloudy models. We overlay the temperature histograms of 3-band fits on the histograms of 4-band fits in Fig. 5. The properties of 3-band fits for both fields are listed in Table 2. For
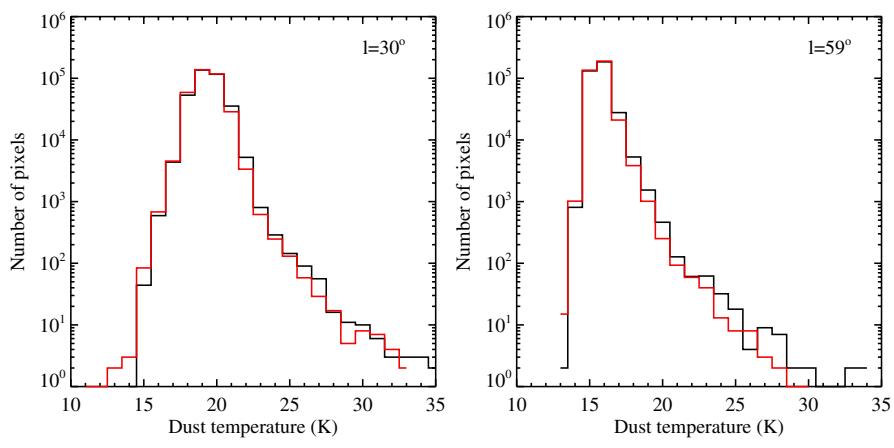

Fig. 5. Comparison of 3-band and 4-band fits for Cloudy models. Left panel: the histograms of the $l=30^{\circ}$ field. Right panel: the histograms of the $l=59^{\circ}$ field. Black line is the result of 3-band fits for Cloudy models; red line is the result of 4-band fits for Cloudy models.

Table 2. Results of 3-band fits for the Cloudy model

\begin{tabular}{lcc}
\hline \hline Fields & $l=30^{\circ}$ & $l=59^{\circ}$ \\
\hline Average $T_{\text {dust }}(\mathrm{K})$ & $18.7^{a}$ & $14.9^{b}$ \\
Max $T_{\text {dust }}(\mathrm{K})$ & 34.3 & 33.6 \\
Min $T_{\text {dust }}(\mathrm{K})$ & 13.7 & 12.9 \\
\hline
\end{tabular}

Notes. ${ }^{(a)}$ The RMS is $1.1 \mathrm{~K} .{ }^{(b)}$ The RMS is $0.5 \mathrm{~K}$.
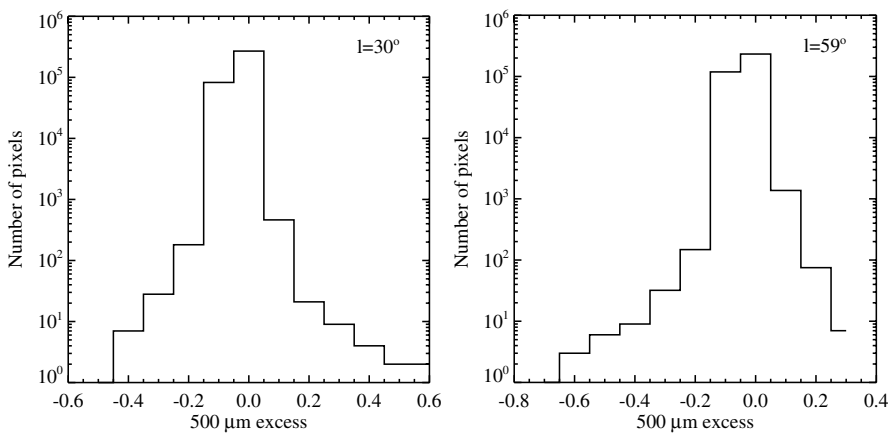

Fig. 6. $500 \mu \mathrm{m}$ excess. Left panel: excess histograms of the $l=30^{\circ}$ field. Right panel: excess histograms of the $l=59^{\circ}$ field.

both fields, part of histograms of 3-band fits and 4-band fits are overlapped.

For the $l=30^{\circ}$ field, $99.5 \%$ of all pixels of 3-band fits are consistent with 4-band fits within the confidence interval of 4-band fits. For the $l=59^{\circ}$ field, $98.3 \%$ of all pixels of 3-band fits are consistent with 4-band fits within the confidence interval. The high degree of consistency indicates that $500 \mu \mathrm{m}$ data point has little influence on the results of SED fitting, and there is no significant excess of a $500 \mu \mathrm{m}$ emission. More specifically, we calculate the $500 \mu \mathrm{m}$ excess by the equation,

excess $=\frac{F_{500}^{\mathrm{obs}}-F_{500}^{\text {model }}}{F_{500}^{\mathrm{obs}}}$,

where $F_{500}^{\text {obs }}$ is the observed $500 \mu \mathrm{m}$ intensity and $F_{500}^{\text {model }}$ is the model predicted intensity. Histograms of the $500 \mu \mathrm{m}$ excess is presented in Fig. 6. The pixel distribution of excess for both fields skew toward positive values, and the average excess with and without negative values is $1 \%$ and $2 \%$ for both fields. Thus, there is no detection of any notable $500 \mu \mathrm{m}$ excess in the Hi-GAL SDP fields.

Our calculation of the $500 \mu \mathrm{m}$ excess is consistent with the results of Paradis et al. (2012). With respect to the predictions of 


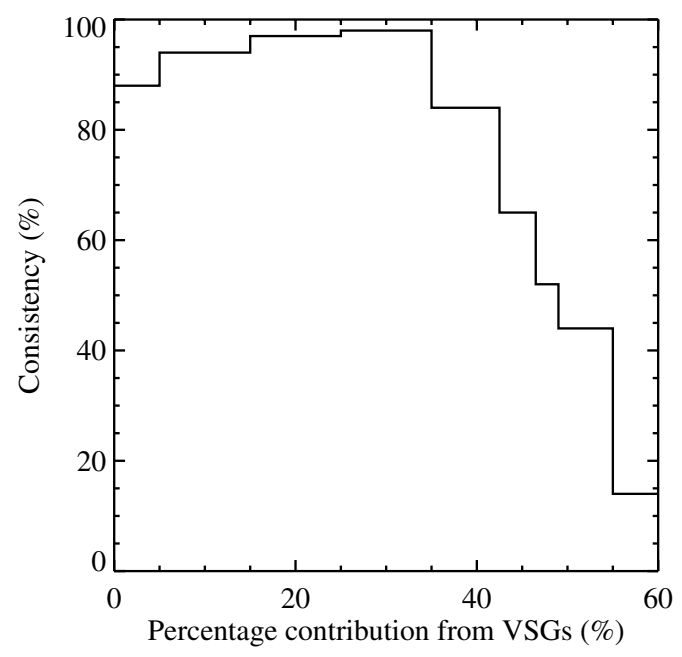

Fig. 7. Consistency of 5-band and 4-band fits for Cloudy models.

a modified black-body model, Paradis et al. (2012) reported that the $500 \mu \mathrm{m}$ excess in the peripheral parts of the Galactic plane can be up to $16 \%$ to $20 \%$. Nevertheless, they found the median value of the $500 \mu \mathrm{m}$ excess is only $1 \%$ and $3 \%$ in the $l=30^{\circ}$ field and the $l=59^{\circ}$ field, respectively.

\section{The VSG contribution to the $70 \mu \mathrm{m}$ intensity}

Based on the results of 4-band fits for Cloudy models, we include the $70 \mu \mathrm{m}$ data in the SED fitting to estimate the VSG contribution to the $70 \mu \mathrm{m}$ intensity. In the 5-band fits, the scaling factor $s$ in Eq. (2) is the same as in the 4-band fits for Cloudy models, and $G_{0}$ is the only free parameter.

Since the VSG contributes to the $70 \mu \mathrm{m}$ emission, we should subtract the contribution from the VSG when we fit the SED using 5-band data to derive the equilibrium temperature of the BGs. We assume that VSGs contribute $x \%$ to the $70 \mu \mathrm{m}$ intensity. After subtracting $x \%$ of the $70 \mu \mathrm{m}$ intensity coming from the VSGs, we fit the SED using 5-band data.

We compare the results of 5-band fits with 4-band fits for $x=0,10,20,30,40,45,48,50$, and 60 , and derive the consistency, or the percentage of pixels whose temperature of 5-band fits are consistent with 4-band fits within the $68 \%$ confidence interval of 4-band fits. Since about $10 \%$ of all pixels in the $l=59^{\circ}$ field have negative values of the $70 \mu \mathrm{m}$ emission, which might be caused by improper calibration in this waveband, we only analyze the $l=30^{\circ}$ field here.

The histogram of consistency is presented in Fig. 7. The value of consistency increases from $88 \%$ to $98 \%$ when the VSG contribution increases from $0 \%$ to $30 \%$. When the VSG contribution is more than $30 \%$, the value of consistency decreases with the increasing contribution. The consistency is $52 \%$ and $44 \%$ when the contribution is $48 \%$ and $50 \%$, respectively. Thus, the
VSG contribution to the $70 \mu \mathrm{m}$ intensity can be from $0 \%$ up to $50 \%$. The temperature distributions of the 5-band fits are most consistent (consistency $>95 \%$ ) with the 4-band fits when the contribution is $20 \%$ to $30 \%$. Our estimation of $70 \mu \mathrm{m}$ contribution from the VSGs is consistent with Compiègne et al. (2010), who found that the VSG contribution goes from $10 \%$ up to $50 \%$ with a median value of $27 \%$ in a diffuse region of the $l=59^{\circ}$ field.

\section{Summary}

We derive temperature maps of the BGs in the fields of $l=30^{\circ}$ and $l=59^{\circ}$ by considering radiative transfer and grain physics at the same time. The dust temperatures of the $l=30^{\circ}$ field range from a minimum $10.9 \mathrm{~K}$ in diffuse regions to a maximum $32.8 \mathrm{~K}$ with an average value of $18.7 \pm 0.9 \mathrm{~K}$. For the $l=59^{\circ}$ field, the dust temperatures range from $12.8 \mathrm{~K}$ to $29.4 \mathrm{~K}$ with an average value of $15.2 \pm 0.6 \mathrm{~K}$. Compared to results for dust-only models, we find that the radiative transfer affects the FIR SED and makes the temperature distribution shift toward higher value of temperature.

We explore the $500 \mu \mathrm{m}$ excess by fitting the dust SED without the $500 \mu \mathrm{m}$ waveband and find that there is no significant excess of a $500 \mu \mathrm{m}$ emission in the two SDP fields. The SED fitting of 5-band data is performed to estimate the VSG contribution to the $70 \mu \mathrm{m}$ intensity. The VSGs can contribute up to $50 \%$ of the observed $70 \mu \mathrm{m}$ intensity.

Acknowledgements. We thank the anonymous referee for helpful comments. This study is supported by funding KJCX2-YW-T20 of Chinese Academy of Science.

\section{References}

Abel, N. P., van Hoof, P. A. M., Shaw, G., Ferland, G. J., \& Elwert, T. 2008, ApJ, 686,1125

Avni, Y., \& Bahcall, N. A. 1976, ApJ, 209, 16

Bernard, J. -P., Paradis, D., Marshall, D., et al. 2010, A\&A, 518, L88

Black, J. H. 1987, Astrophys. Space Sci. Lib., 134, 731

Boulanger, F., Abergel, A., Bernard, J. -P., et al. 1996, A\&A, 312, 256

Compiègne, M. 2010, ASP Conf. Ser., 438, 55

Compiègne, M., Flagey, N., Noriega-Crespo, A., et al. 2010, ApJ, 724, L44

Draine, B. T. 2003, ARA\&A, 41, 241

Elia, D., Molinari, S., Fukui, Y., et al. 2013, ApJ, 772, 45

Ferland, G. J., Porter, R. L., van Hoof, P. A. M., et al. 2013, Rev. Mex. Astron. Astrophys., 49, 137

Fitzpatrick, E. L. 2004, in Astrophysics of Dust, eds. A. N. Witt, G. C. Clayton, \& B. T. Draine (San Francisco, CA: ASP), ASP Conf. Ser. 309, 33

Griffin, M. J., Paladini, R., Noriega-Crespo, A., et al. 2010, A\&A, 518, L3

Habing, H. J. 1968, Bull. Astron. Inst. Netherlands, 19, 421

Mathis, J. S., Rumpl, W., \& Nordsieck, K. H. 1977, ApJ, 217, 425

Molinari, S., Riedinger, J. R., \& Passvogel, T. 2010, A\&A, 518, L100

Paradis, D., Veneziani, M., Noriega-Crespoet, A., et al. 2010, A\&A, 520, L8

Paradis, D., Paladini, R., Noriega-Crespo, A., et al. 2012, A\&A, 537, A113

Piazzo, L., Ikhenaode, D., Natoli, P., et al. 2012, IEEE Trans. Image Process., 21,3687

Pilbratt, G. L., Riedinger, J. R., Passvogel, T., et al. 2010, A\&A, 518, L1

Poglitsch, A., Waelkens, C., Geis, N., et al. 2010, A\&A, 518, L2 